\title{
Pitte Jean-Robert, Dictionnaire amoureux de la
}

Bourgogne

Paris, Plon, 2014, 682 p.

Philippe Boulanger

\section{(2) OpenEdition}

Journals

Édition électronique

URL : https://journals.openedition.org/geohist/1359

DOI : 10.4000/geohist.1359

ISSN : 2264-2617

Éditeur

Association française de la Revue de géographie historique

Référence électronique

Philippe Boulanger, « Pitte Jean-Robert, Dictionnaire amoureux de la Bourgogne », Revue de géographie historique [En ligne], Comptes-rendus, mis en ligne le 20 novembre 2015, consulté le 12 juin 2021.

URL : http://journals.openedition.org/geohist/1359; DOI : https://doi.org/10.4000/geohist.1359

Ce document a été généré automatiquement le 12 juin 2021.

\section{cc)}

$\mathrm{Ce}(\mathrm{tte})$ œuvre est mise à disposition selon les termes de la Licence Creative Commons Attribution -

Pas d'Utilisation Commerciale - Pas de Modification 4.0 International. 


\section{Pitte Jean-Robert, Dictionnaire amoureux de la Bourgogne}

Paris, Plon, 2014, 682 p.

Philippe Boulanger

\section{RÉFÉRENCE}

Pitte Jean-Robert, Dictionnaire amoureux de la Bourgogne, Paris, Plon, 2014, 682 p.

1 Le dernier ouvrage de Jean-Robert Pitte, professeur de géographie, ancien président de l'Université Paris-Sorbonne et membre de l'Institut, est l'un des plus remarquables et surprenants. Chacun de ses livres pouvait déjà nous étonner. Celui-ci ne manque pas de nous enchanter à chaque page. Nous y apprenons beaucoup sur la Bourgogne que l'auteur connaît à la fois par sa propre expérience personnelle, qu'il nous fait partager de manière intime comme un guide nous conte les plus belles histoires, et par son immense savoir scientifique de géographe. "Je suis fier d'être bourguignon de cour, d'une province où je ne suis pas né et où n'est sans doute né aucun des mes ancêtres " nous écrit l'auteur, lui-même ayant dirigé quelques années, et habité partiellement, une propriété viticole d'un hectare, à Villars-Fontaine, produisant un vin délectable de Chardonnay. Nous y apprenons beaucoup sur des sujets variés: la romanité antique et les grandes abbayes (Cluny et Cîteaux) du Moyen Age occidental, le rayonnement du grand duché de l'Occident, les reliefs ondulés et la richesse de des paysages de Bourgogne (Chablis, Charolais, Mâconnais, le Morvan, etc.), les sites les plus significatifs (Fontenay, Dijon, Beaune, Joigny, le Creusot, etc.), les Bourguignons dont la personnalité est «faite de franchise, de truculence, de distance vis-à-vis d'eux-mêmes ", la richesse gastronomique et les divers vignobles qui produisent les plus grands vins du monde, les personnalités les plus illustres (Bernard de Clairvaux, Jacques-Bénigne Bossuet, Alphonse de Lamartine, Colette, etc.) ou moins connues (le chanoine Kir, Charles de Brosses, Gaston Gérard, etc.). 
2 Jean-Robert Pitte donne des clefs de lecture qu'aucun ouvrage ne pouvait nous livrer ainsi. Il faut dire qu'il a parcouru, dans toutes les dimensions, cette région si riche par son histoire, sa culture et sa géographie. "Des années après l'éblouissement de Chorey, mon addiction à la Bourgogne s'est ensuite ancrée dans la Côte de Nuits, entre le Clos de Vougeot, Vosne-Romanée et Nuits-Saint-Georges, et dans la montagne qui la domine, les Hautes-Côtes de Nuits, au pied de la colline inspirée de Vergy, sur le coteau ensoleillé de Villars-Fontaine. Dionysos est né deux fois; moi aussi, et ma cuisse de Zeus s'appelle la Bourgogne». Le Dictionnaire amoureux de la Bourgogne est donc un ouvrage à la fois personnel, autobiographique pour certains passages, et savant dans un style propre à l'auteur, vif et alerte, un mélange d'histoires personnelles et vécues, de sensations ressenties depuis ses premières escapades de géographe, une sensibilité d'homme du terroir et du monde urbain, un regard épanoui et réellement amoureux pour les hommes qui la composent.

3 L'ouvrage est donc savoureux parce qu'il n'est pas académique et ne ressemble nullement à ce que nous prenons l'habitude de découvrir sur une région. Nous ne nous lassons pas d'apprendre à chaque page. Il se compose d'une centaine d'entrées, toutes différentes par leur nature. La diversité des thèmes et des approches, menés avec un corps vibrant et un cœur battant, aborde aussi bien le site d'Alésia que les villes de Dijon et de Beaune, les paysages, les vignobles et les villages de Meursault, Chambertin, Nuits-Saint-Georges ou de Vosne-Romanée, que les escargots, le cassis, la cocotte, le cornichon et la bouteille de Bourgogne, l'accent bourguignon (le " $\mathrm{r} »)$ et les climats que la vie de Jean-Philippe Rameau et de Vauban, ses rivières (Saône, Seine, Yonne) que son jambon persillé et sa moutarde. Jean-Robert Pitte nous conduit dans une autre sphère avec passion et le plaisir de la partager. Il est plus qu'un ouvrage qui vous donne envie de visiter ou revisiter la région. Il vous la fait découvrir et aimer de manière inattendue, par ses richesses matérielles et immatérielles. 